\title{
Solução do problema de Riemann para escoamento de água-óleo na presença de solvente em meio poroso.
}

\author{
Yvonne Santa Cruz Cárdenas ${ }^{1}$ \\ LAMAP, UFJF, Juiz de Fora, MG \\ Prof. Dr. Grigori Chapiro ${ }^{2}$ \\ LAMAP, UFJF, Juiz de Fora, MG \\ Dr. Luis Fernando Lozano Guerrero ${ }^{3}$ \\ LAMAP, UFJF, Juiz de Fora, MG
}

Resumo. Este trabalho estuda o sistema de leis de conservação que descreve o modelo simplificado do trabalho de Walsh e Lake (1989). Foi resolvido o problema de Riemann correspondente classificando as possíveis soluções de acordo com a saturação de água no ponto de injeção. Foram encontradas três possibilidades: (1) onda de choque, (2) onda de rarefação, (3) ondas compostas. Foi demonstrado que a onda de choque é entrópica. Para isso, foi provado que a existência da solução na forma de ondas viajantes do problema viscoso associado conectando os equilíbrios correspondentes aos estados do problema de Riemann na forma de $\alpha$ e $\omega$-limites. Todos os resultados teóricos foram validados através de simulações numéricas.

Palavras-chave. Leis de conservação. Ondas viajantes. Problema de Riemann. Injeção de solventes em meios porosos. Recuperação avançada de petróleo.

\section{Introdução}

A injeção de solventes é uma técnica de recuperação avançada de petróleo. É um dos primeiros métodos de produção de óleo adicional que melhora a mobilidade do fluido deslocado, implicando num aumento do fator de recuperação [1]. Em [2] é mostrado um sistema de leis de conservação que estuda o deslocamento de óleo por um solvente miscível na fase aquosa imiscível. O fluxo fracionário de água é considerado como uma função dependendo da saturação de água e do volume fracionário de solvente na fase oleica, entre outras considerações. Os autores mostram um procedimento gráfico para determinar a solução, encontram uma taxa ótima de água com solvente entre outros.

Neste trabalho estudamos uma versão mais simples do modelo mostrado em [2], considerando o fluxo fracionário de água só como função da saturação de água. Provamos a existência da solução na forma de ondas viajantes. Resolvemos o problema de Riemann fixando o estado mais (ou condição inicial) e variando o estado menos (condição de injeção). Verificamos os resultados numericamente usando MATLAB e RCD (Reaction-Convection-Diffusion Equations' Solver), ver [3] para detalhes.

\section{Solução na forma de ondas viajantes}

Em [2], um sistema de leis de conservação é dado e o estudamos num caso particular considerando o fluxo fracionário da água como uma função que só depende da saturação da água:

$$
\partial_{t} S_{w}+\partial_{x} f_{w}=0, \quad \partial_{t}\left(C_{s o} S_{o}\right)+\partial_{x}\left(C_{s o} f_{o}\right)=0,
$$

\footnotetext{
${ }^{1}$ yvonne@ice.ufjf.br

2 grigori@ice.ufjf.br

3 luisfer99@gmail.com
} 
onde $S_{w}$ é a saturação de água, $S_{o}$ é a saturação de óleo, $C_{s o}$ é o volume fracionário de solvente na fase oleica, $f_{w}$ é o fluxo fracionário da água e $f_{o}$ é o fluxo fracionário do óleo, definidos por:

$$
f_{w}+f_{o}=1, \quad f_{w}\left(S_{w}\right)=\left(K_{r w} / \mu_{w}\right)\left(K_{r w} / \mu_{w}+K_{r o} / \mu_{o}\right)^{-1},
$$

onde $\mu_{w}$ e $\mu_{o}$ são viscosidades constantes, dadas na Tabela 1. As permeabilidades relativas da fase aquosa $K_{r w}$ e da fase oleica $K_{r o}$ são funções de $S_{w}$ como definidas a seguir

$$
K_{r w}\left(S_{w}\right)=\left\{\begin{array}{l}
0, S_{w}<S_{w c}, \\
S^{2}, S_{w c}<S_{w} \leq 1,
\end{array} \quad K_{r o}\left(S_{w}\right)=\left\{\begin{array}{l}
0, S_{w} \geq 1-S_{o r}, \\
(1-S)^{2}, 0 \leq S_{w} \leq 1-S_{o r},
\end{array}\right.\right.
$$

onde $S=\left(1-S_{w}-S_{o r}\right) /\left(1-S_{w c}-S_{o r}\right), S_{w c}$ e $S_{o r}$ são a saturação de água conata e a saturação do óleo residual, respectivamente, e são dadas na Tabela 1. O meio poroso é considerado saturado, i.e. $S_{w}+S_{o}=1$. Consideramos o Sistema (1) com a seguinte condição inicial

Tabela 1: Valores constantes para os parâmetros.

\begin{tabular}{cccl}
\hline Símbolo & Descrição & Valor & Fonte \\
\hline$S_{w c}$ & Saturação de água conata & 0.0 & {$[2]$} \\
$S_{o r}$ & Saturação de óleo residual & 0.15 & {$[2]$} \\
$\mu_{w}$ & Viscosidade da fase água & 1 & {$[2]$} \\
$\mu_{o}$ & Viscosidade da fase óleo & 2 & {$[2]$} \\
$S_{w}^{+}$ & Saturação de água no ponto inicial & 0.75 & \\
$f_{w}\left(S_{w}^{+}\right)$ & Fluxo fracionário avaliado em $S_{w}^{+}$ & 0.9911894273 & Eq.(2) \\
\hline
\end{tabular}

$$
S_{w}(x, 0)=\left\{\begin{array}{l}
S_{w}^{-}, x<0, \\
S_{w}^{+}, x>0,
\end{array} \quad \text { e } \quad C_{s o}(x, 0)=\left\{\begin{array}{l}
C_{s o}^{-}, x<0 \\
C_{s o}^{+}, x>0
\end{array}\right.\right.
$$

onde $U^{-}=\left(S_{w}^{-}, C_{s o}^{-}\right)$é o estado menos e $U^{+}=\left(S_{w}^{+}, C_{s o}^{+}\right)$é o estado mais. Optamos por estudar os choques que têm perfil viscoso, portanto, são adicionados termos de viscosidade ao Sistema (1):

$$
\partial_{t} S_{w}+\partial_{x} f_{w}=\epsilon \partial_{x x} S_{w}, \quad \partial_{t}\left(C_{s o} S_{o}\right)+\partial_{x}\left(C_{s o} f_{o}\right)=\epsilon \partial_{x x} C_{s o}
$$

onde $\epsilon$ é uma constante não nula. Isto permite encontrar a solução na forma de ondas viajantes do sistema associado, trocando as variáveis de $(x, t)$ para coordenadas viajantes $(\xi=x-v t, t)$, onde $v$ representa a velocidade da onda viajante. O sistema original de equações diferenciais parciais (EDPs) pode ser reescrito como um sistema de equações diferenciais ordinárias (EDOs), similar ao que foi feito em [5]. Além disso, os estados menos e mais correspondem aos seguintes pontos de equilíbrio esquerdo e direito:

$$
\lim _{\xi \rightarrow-\infty} U(\xi)=U^{-}, \lim _{\xi \rightarrow+\infty} U(\xi)=U^{+} .
$$

Ao fazer a mudança de variáveis para coordenadas viajantes obtém-se o sistema de EDOs

$$
\left\{\begin{array}{l}
d_{\xi} S_{w}=\epsilon^{-1}\left(f_{w}-f_{w}^{+}-v\left(S_{w}-S_{w}^{+}\right)\right)=F_{1}\left(S_{w}, C_{s o}\right), \\
d_{\xi} C_{s o}=\epsilon^{-1}\left(\left(1-f_{w}\right) C_{s o}-\left(1-f_{w}^{+}\right) C_{s o}^{+}-v\left(\left(1-S_{w}\right) C_{s o}-\left(1-S_{w}^{+}\right) C_{s o}^{+}\right)\right)=F_{2}\left(S_{w}, C_{s o}\right) .
\end{array}\right.
$$

\subsection{Localização dos pontos de equilíbrio}

Provar que o Problema de Riemann (1), (4) tem solução na forma de ondas viajante significa provar que o Sistema (7) tem uma órbita que liga o equilíbrio esquerdo ao direito. Para localizar os equilíbrios de (7) estudaremos os zeros do campo vetorial associado:

$$
d_{\xi} S_{w}=0, d_{\xi} C_{s o}=0 \text {, onde } v=\left(f_{w}^{+}-f_{w}^{-}\right) /\left(S_{w}^{+}-S_{w}^{-}\right) .
$$


Observa-se que para cada $S_{w}^{-}$tem-se um valor diferente para $v$. Usando os valores dados na Tabela 1, pode ser obtido a curva de nível de $F_{2}\left(S_{w}, C_{s o}\right)=0$ concluindo o seguinte:

- Os pontos de equilíbrio $\left(S_{w}, C_{s o}\right)$ encontram-se sobre uma reta horizontal.

- As segundas componentes dos pontos de equilíbrio devem ser iguais a 1 , i.e. $C_{\text {so }}=1$.

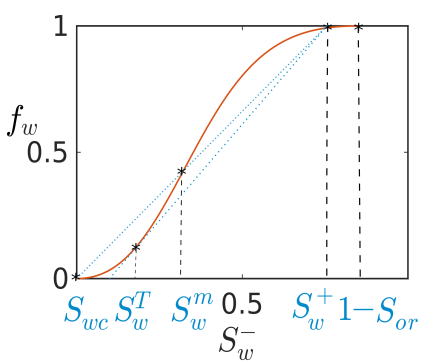

(a) Fluxo fracionário da água (vermelha) intersectado por duas retas pontilhadas (celeste).

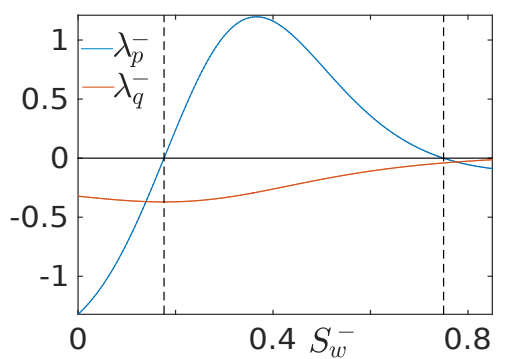

(b) Autofunções associadas $\left(S_{\bar{w}}^{-}, C_{s o}^{-}\right)$.

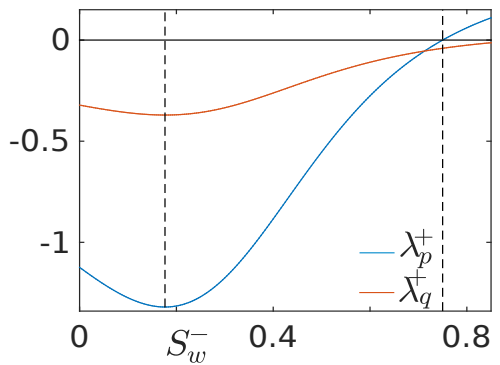

a (c) Autofunções associadas a $\left(S_{w}^{+}, C_{s o}^{+}\right)$

Figura 2.1: Equilíbrios de (7), com $S_{w}^{+}=0.75$. (a) Os pontos $\left(S_{w}^{T}, f_{w}\left(S_{w}^{T}\right)\right)$ e $\left(S_{w}^{m}, f_{w}\left(S_{w}^{m}\right)\right)$ são as interseções entre $f_{w}$ e a tangente a $f_{w}$ que passa por $\left(S_{w}^{+}, f_{w}^{+}\right)$e a reta que liga $\left(S_{w c}, f_{w}\left(S_{w c}\right)\right)$ e $\left(S_{w}^{+}, f_{w}^{+}\right)$. (b) e (c) Classificação dos pontos de equilíbrio.

Para ter informação sobre $S_{w}$ consideramos $F_{1}\left(S_{w}, C_{s o}\right)=0$, como $\epsilon \neq 0$ obtém-se:

$$
f_{w}=v S_{w}+\left(f_{w}^{+}-v S_{w}^{+}\right)
$$

Esta equação representa os pontos de interseção de uma reta de inclinação $v$ com a curva de fluxo fracionário da água nos pontos $\left(S_{w}^{+}, f_{w}^{+}\right)$e $\left(S_{w}, f_{w}\right)$, veja Fig. 2.1(a). Na Fig. 2.1(a) mostra-se, que dependendo do valor de $S_{w}$ tem-se dois ou três pontos de interseção.

\section{$2.2 \quad$ Número de pontos de equilíbrio}

Para identificar os possíveis equilíbrios estudaremos a primeira componente do campo (8). Na Eq. (9), a reta $y=v S_{w}+\left(f_{w}^{+}-v S_{w}^{+}\right)$tem uma inclinação $v$ que depende de $S_{w}^{-}$, veja (8). A variação de $v$ cria um leque de retas que intersectam a função do fluxo fracionário da água. Por exemplo, na Fig. 2.1(a) tem-se duas retas pontilhadas (cor celeste) que intersectam $f_{w}$. Uma tem inclinação $\left(f_{w}\left(S_{w}^{+}\right)-f_{w}\left(S_{w}^{T}\right)\right) /\left(S_{w}^{+}-S_{w}^{T}\right)$ e é tangente à curva de fluxo fracionário da água no ponto $S_{w}^{T}$. A outra passa pela origem e por $\left(S_{w}^{+}, f_{w}\left(S_{w}^{+}\right)\right)$, intersectando a curva $f_{w}$ em 3 pontos: $\left(S_{w c}, f_{w}\left(S_{w c}\right)\right),\left(S_{w}^{m}, f_{w}\left(S_{w}^{m}\right)\right),\left(S_{w}^{+}, f_{w}\left(S_{w}^{+}\right)\right)$. O valor de $S_{w}^{T}$ é calculado com a seguinte equação:

$$
f_{w}^{\prime}\left(S_{w}^{T}\right)=\left(f_{w}\left(S_{w}^{+}\right)-f_{w}\left(S_{w}^{T}\right)\right) /\left(S_{w}^{+}-S_{w}^{T}\right) .
$$

Entretanto, o valor de $S_{w}^{m}$ obtém-se calculando $f_{w}\left(S_{w}^{m}\right)=\theta_{2} S_{w}^{m}$, onde $\theta_{2}$ é a inclinação da reta que passa pela origem e pelo ponto $\left(S_{w}^{+}, f_{w}^{+}\right)$, veja Fig. 2.1(a). Com o estudo geométrico feito na Fig. 2.1(a) é demonstrada a Proposição 2.1.

Proposição 2.1. A reta $y=v S_{w}+\left(f_{w}^{+}-v S_{w}^{+}\right)$da Eq. (9) pode intersectar a curva do fluxo fracionário da água em dois ou três pontos, obtendo o número de equilíbrios.

- Se $\left.S_{w}^{-} \in\right] S_{w}^{+}, 1-S_{\text {or }}$, existem dois equilíbrios: $\left(S_{w}^{-}, C_{s o}^{-}\right)$e $\left(S_{w}^{+}, C_{s o}^{+}\right)$.

- Se $\left.\left.S_{w}^{-} \in\right] S_{w}^{m}, S_{w}^{+}\right]$, existem dois equilíbrios: $\left(S_{w}^{-}, C_{s o}^{-}\right)$e $\left(S_{w}^{+}, C_{s o}^{+}\right)$.

- $S e S_{w}^{-} \in\left[S_{w}^{T}, S_{w}^{m}\right]$ existem três equilíbrios: $\left(S_{w}^{e}, C_{s o}^{e}\right),\left(S_{w}^{-}, C_{s o}^{-}\right),\left(S_{w}^{+}, C_{s o}^{+}\right)$, tal que $S_{w}^{e}<$ $S_{w}^{-}<S_{w}^{+}$e $S_{w}^{e} \in\left[S_{w c}, S_{w}^{T}[\right.$. 
- Se $S_{w}^{-} \in\left[S_{w c}, S_{w}^{T}\left[\right.\right.$ existem três equilíbrios: $\left(S_{w}^{-}, C_{s o}^{-}\right),\left(S_{w}^{e}, C_{s o}^{e}\right),\left(S_{w}^{+}, C_{s o}^{+}\right)$, tal que $S_{w}^{-}<$ $S_{w}^{e}<S_{w}^{+}$e $\left.\left.S_{w}^{e} \in\right] S_{w}^{T}, S_{w}^{m}\right]$.

Observação 2.1. A Proposição 2.1 vale para toda função de fluxo fracionário em forma de $S$.

\subsection{Classificação dos pontos de equilíbrio}

Na seção anterior determinamos o número de equilíbrios que tem o Sistema (7) dependendo da posição de $S_{w}^{-}$. Nesta seção, os classificamos nas vizinhanças correspondentes com base no Teorema de Hartman-Grobman. Considere o Sistema (7) linearizado $U^{\prime}=J\left(U_{0}\right) U$, onde $U=\left(S_{w}, C_{s o}\right)^{T}$ e $J\left(U_{0}\right)$ é a matriz jacobiana avaliada no ponto de equilíbrio $U_{0}=\left(S_{w_{0}}, C_{s o_{0}}\right)$. Calculamos os autovalores obtendo as raízes $\operatorname{de} \operatorname{det}(J(U)-\lambda I)=0$ :

$$
\lambda_{p}=\epsilon^{-1}\left(f_{w}^{\prime}\left(S_{w}\right)-v\right) \text { e } \lambda_{q}=\epsilon^{-1}\left(-v\left(1-S_{w}\right)+1-f_{w}\right),
$$

com seus correspondentes autovetores:

$$
\omega_{p}=\left[\begin{array}{ll}
a & 1
\end{array}\right], \omega_{q}=\left[\begin{array}{ll}
0 & 1
\end{array}\right] \text {, onde, } a=\epsilon\left(\lambda_{q}-\lambda_{p}\right) /\left(-C_{s o} \lambda_{p}\right) .
$$

No que segue os super-índices $(-)$ e $(+)$ denotam as autofunções associadas aos equilíbrios esquerdo e direito, respectivamente. As autofunções são definidas como:

$$
\left\{\begin{array}{l}
\lambda_{p}^{\mp}=\lambda_{p}\left(S_{w}^{\mp}, C_{s o}^{\mp}\right)=\epsilon^{-1}\left(f_{w}^{\prime}\left(S_{w}^{\mp}\right)-v\right), \\
\lambda_{q}^{\mp}=\lambda_{q}\left(S_{w}^{\mp}, C_{s o}^{\mp}\right)=\epsilon^{-1}\left(1-f_{w}\left(S_{w}^{\mp}\right)-v\left(1-S_{w}^{\mp}\right)\right) .
\end{array}\right.
$$

Com os valores da Tabela 1 obtemos os gráficos das autofunções definidas em (13), veja as Figs. 2.1(b) e 2.1(b). Estas gráficas representam a classificação dos autovalores associados aos equilíbrios esquerdo e direito. Observando a mudança de sinal nestas figuras é demonstrado Teorema 2.1.

Observação 2.2. Ao calcular $\lambda_{p}^{\mp}=0$ e $\lambda_{q}^{\mp}=0, \epsilon$ não influencia no sinal das autofunções.

Teorema 2.1. Seja o Sistema de EDOs (7) $\operatorname{com}\left(S_{w}^{+}, C_{s o}^{+}\right)=(0.75,1)$ e as autofunções $\left(\lambda_{p}^{\mp}, \lambda_{q}^{\mp}\right)$ descritas em (13). Com $S_{w}^{T}$ dado em (10), os equilíbrios são classificados como segue:

- Se $S_{w}^{-} \in\left[S_{w c}, S_{w}^{T}[\right.$, têm-se três equilíbrios: o esquerdo e direito são atratores hiperbólicos e o extra é uma sela hiperbólica (não há possibilidade de conexão).

- $\left.\left.S_{w}^{-} \in\right] S_{w}^{T}, S_{w}^{m}\right]$, têm-se três equilíbrios: o esquerdo é uma sela hiperbólica, o direito é um atrator hiperbólico e o extra é um atrator hiperbólico.

- $\left.S_{w}^{-} \in\right] S_{w}^{m}, S_{w}^{+}[$, têm-se dois equilíbrios: o esquerdo é uma sela hiperbólica e o direito é um atrator hiperbólico.

- Se $\left.S_{w}^{-} \in\right] S_{w}^{+}, 1-S_{\text {or }}$ [, têm-se dois equilíbrios: o esquerdo é um atrator hiperbólico e o direito é uma sela hiperbólica (não há possibilidade de conexão).

Teorema 2.2. Seja o Sistema (7) com v definido em (8), $\left(S_{w}^{+}, C_{\text {so }}^{+}\right)=(0.75,1), C_{\text {so }}^{-}=1 e$ $\left.S_{w}^{-} \in\right] S_{w}^{T}, S_{w}^{+}\left[\right.$, onde $S_{w}^{T}$ é como em (10). Com os valores da Tabela 1, existe a solução deste sistema $\left(S_{w}(\xi), C_{\text {so }}(\xi)\right)$, tal que $\lim _{\xi \rightarrow-\infty}\left(S_{w}(\xi), C_{\text {so }}(\xi)\right)=\left(S_{w}^{-}, 1\right)$ e $\lim _{\xi \rightarrow \infty}\left(S_{w}(\xi), C_{\text {so }}(\xi)\right)=(0.75,1)$.

Demonstração. Se $\left.S_{w}^{-} \in\right] S_{w}^{T}, S_{w}^{+}\left[\right.$tem-se duas possibilidades: ou $\left.\left.S_{w}^{-} \in\right] S_{w}^{T}, S_{w}^{m}\right]$, ou $S_{w}^{-} \in$ ]$S_{w}^{m}, S_{w}^{+}[$, veja Fig. 2.2(a).

a) Se $\left.S_{w}^{-} \in\right] S_{w}^{m}, S_{w}^{+}[$, pelo Teor. 2.1 só existem dois equilíbrios: o esquerdo $(L)$ uma sela hiperbólica e o direito $(R)$ um atrator hiperbólico.

Como $L$ é uma sela hiperbólica, $L$ tem duas variedades invariantes. A reta amarela vertical que passa pelo equilíbrio $R$ é uma variedade invariante, veja Fig. 2.2(b). Portanto, as retas verticais 
passando pelos pontos $L$ e $R$ são variedades invariantes. Isto implica que a região entre elas $\Omega=\left\{\left(S_{w}, C_{s o}\right): S_{w}^{-}<S_{w}<S_{w}^{+}\right\}$é um conjunto invariante pelo Sistema (7).

Suponhamos, por absurdo, que não exista uma órbita que ligue $L$ a $R$ como no enunciado do teorema. Parte da variedade instável de $L$ fica à direita de $L$ como indicado em verde na Fig. 2.2(b). Esta variedade, que chamamos $U^{u}(L)$, é uma órbita do Sistema (7). Sem perda de generalidade suponhamos que esta órbita cresce na direção positiva de $C_{s o}$ quando varia $\xi$, veja Fig. 2.2(b). Para todos os pontos $\left(S_{w}^{+}, C_{s o}\right)$ com $C_{\text {so }}$ positivo, podemos traçar o menor segmento de reta horizontal que junta $U^{u}(L)$ à reta $S_{w}=S_{w}^{+}$. Note que o campo vertical no extremo esquerdo deste segmento é positivo, enquanto que no extremo direito é negativo. Pelo Teorema de Valor Médio, existe ao menos um ponto neste segmento horizontal tal que o campo vertical nele é nulo. Com isso mostramos que o conjunto de todos os pontos da região $\Omega$ com os pontos onde o campo vertical é nulo não é limitado na direção positiva de $C_{s o}$.

Por outro lado, o conjunto de todos os pontos da região $\Omega$ com os pontos onde o campo vertical é nulo coincide com o conjunto de todos os zeros de $F_{2}\left(S_{w}, C_{s o}\right)$. Considerando os valores dados na Tabela 1 , substituindo $(2)(\operatorname{com}(3))$ em $F_{2}\left(S_{w}, C_{s o}\right)=0$ obtém-se:

$$
F_{2}\left(S_{w}, C_{s o}\right)=\left(C_{s o}\left(a_{1} S_{w}^{2}+a_{2} S_{w}+a_{3}+a_{4} S_{w}^{3}\right)+a_{5} S_{w}^{2}+a_{6} S_{w}+a_{7}\right) /\left(a_{8} S_{w}^{2}+a_{9} S_{w}+a_{10}\right),
$$

onde $a_{i}, \forall i=1, \cdots, 10$ são constantes e é uma função racional com denominador não nulo para $S_{w} \in\left[S_{w c}, 1-S_{o r}\right]$, que em particular se satisfaz para $\left.S_{w} \in\right] S_{w}^{m}, S_{w}^{+}[$. Da Eq. (14) tem-se

$$
C_{s o}\left(S_{w}\right)=-\left(b_{1} S_{w}{ }^{2}-b_{2} S_{w}+b_{3}\right) /\left(b_{4} S_{w}{ }^{3}-b_{5} S_{w}{ }^{2}+b_{6} S_{w}-b_{7}\right)
$$

onde $b_{i} \forall i=1, \cdots, 7$ são constantes e o denominador é não nulo para $S_{w} \in \Omega$. A função $C_{s o}\left(S_{w}\right)$ representa a curva de nível $c_{2}$ de $F_{2}\left(S_{w}, C_{s o}\right)=0$. O gráfico $c_{2}$ é uma curva limitada, dado que $c_{2}$ é contínua definida num intervalo compacto. Isto é uma contradição, pois tínhamos construído um subconjunto ilimitado de $c_{2}$. Portanto, existe a órbita que liga $L$ a $R$.

b) Se $\left.\left.S_{w}^{-} \in\right] S_{w}^{T}, S_{w}^{m}\right]$, pelo Teor. 2.1 tem-se três equilíbrios: o esquerdo $(L)$ é uma sela hiperbólica, o direito $(R)$ é um atrator hiperbólico e o extra $(E)$ é um atrator hiperbólico. Também sabemos que se satisfaz a seguinte relação de ordem $S_{w}^{e}<S_{w}^{T}<S_{w}^{-}<S_{w}^{+}$.

Dado que a variedade estável de $L$ é uma reta vertical (veja reta vermelha na Fig. 2.2(b)) que divide o retrato de fase em duas regiões: à esquerda e à direita dessa reta. O equilíbrio extra fica na região à esquerda da reta. Como esta reta é uma variedade estável logo, como as órbitas formam uma partição do espaço de fase, nenhuma órbita passa pelas duas regiões. Portanto o equilíbrio extra não influencia no estudo da existência de órbita conectando $L$ a $R$. Logo pela parte $a$ ) garantimos a existência da órbita heteroclínica conectando $L$ a $R$.

Na Fig. 2.2(a), as regiões $I, I I$ e $I I I$ mostram onde pode existir (azul) ou não existe solução (vermelha) na forma de ondas viajantes para o Sistema EDPs $(1)$ considerando $\left(S_{w}^{+}, C_{s o}^{+}\right)=(0.75,1)$. Enquanto, na Fig. 2.2(b) as retas vermelha e amarela indicam as variedades invariantes de $L$ e $R$, respectivamente. Estamos supondo que a variedade instável de $L$ (cor verde) explode na direção positiva de $C_{s o}$. Os pontos asteriscos indicam o conjunto solução da Eq. (10).

Corolário 2.1. Seja o Sistema (7) com v definido em (8), $\left(S_{w}^{+}, C_{\text {so }}^{+}\right)=(0.75,1), C_{s o}^{-}=1$, $\left.S_{w}^{-} \notin\right] S_{w}^{T}, S_{w}^{+}\left[\right.$e $S_{w}^{T}$ como em (10). Com os valores da Tabela 1 , não existe a solução $\left(S_{w}(\xi), C_{s o}(\xi)\right.$ do Sistema $(7)$ tal que $\lim _{\xi \rightarrow-\infty}\left(S_{w}(\xi), C_{s o}(\xi)\right)=\left(S_{w}^{-}, 1\right)$ e $\lim _{\xi \rightarrow \infty}\left(S_{w}(\xi), C_{s o}(\xi)\right)=(0.75,1)$.

Teorema 2.3. Seja o Sistema de EDPs (1) com condições iniciais

$$
U^{-}=\left(S_{w}^{-}, 1\right), x<0 \text { e } U^{+}=(0.75,1), x>0 .
$$

Com os valores de parâmetros dados na Tabela 1, existe solução na forma de ondas viajantes para o Problema de Riemann (1), (16), se e somente se, $\left.S_{w}^{-} \in\right] S_{w}^{T}, S_{w}^{+}\left[\right.$e $S_{w}^{T}$ é como (10). 


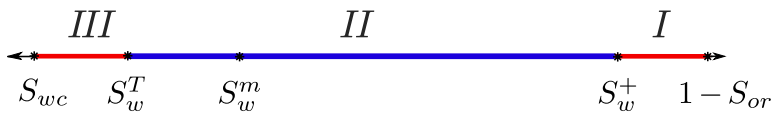

(a) Regiões de $S_{w}^{-}$onde existe e não existe solução do tipo de ondas viajantes.

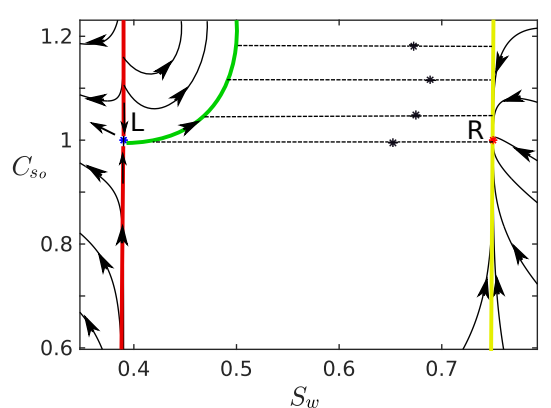

(b) Espaço de Fase de (7) que ajuda na demonstração do Teor. 2.2 .

Figura 2.2: À direita Regiões de $S_{w}^{-}$. À esquerda espaço de fase obtido ao supor que não existe uma órbita heteroclínica que liga $L$ a $R$.

Observação 2.3. O Corolário 2.1 e Teorema 2.3 são demostrados usando os Teoremas 2.1 e 2.2.

Para $\epsilon=1$, na Fig. 2.3(a) mostramos a órbita que liga o equilíbrio esquerdo ao direito e os perfis do Sistema de EDOs (7) são mostrados na Fig. 2.3(b). Os resultados obtidos nesta seção não são influenciados pelo valor de $\epsilon$, mas quando $\epsilon \rightarrow 0$ obtém-se simulações similares.

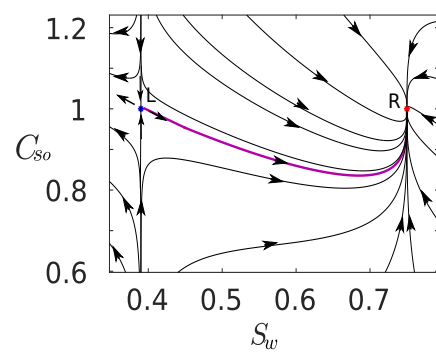

(a) Espaço de fase de (7).

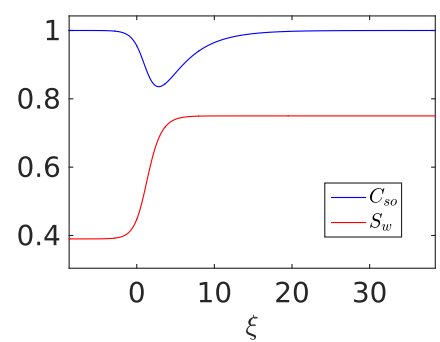

(b) Perfis da solução de (7).

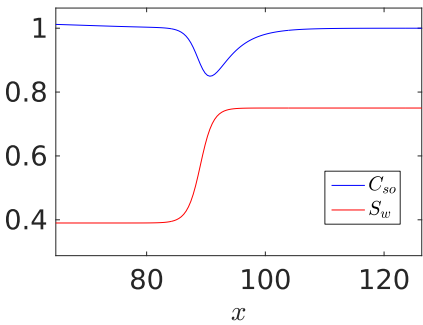

(c) Perfis da solução de (1).

Figura 2.3: Foi considerado $\epsilon=1$ e $U^{-}=(0.39,1)$. Nas Figs. (a) e (b) foi usado MATLAB e na Fig. (c) foi usado RCD.

\section{Ondas de rarefação y Ondas compostas}

Nesta seção verificamos numericamente que o Sistema (1) tem solução na forma de ondas de rarefação e na forma de ondas compostas, para $S_{w}^{-}$que pertence á região $I$ e $I I I$, respectivamente, veja Fig. 2.2(a). Supondo que $U$ é uma função diferenciável de $\xi=x / t$, o Sistema (1) pode ser reescrito como:

$$
U^{\prime}(\xi)=B, \operatorname{com} U^{\prime}(\xi)=\left[\begin{array}{ll}
S_{w}^{\prime} & C_{s o}^{\prime}
\end{array}\right]^{T}, \quad B=\left[1 / f_{w}^{\prime \prime}\left(S_{w}\right) 0\right]^{T} .
$$

A solução do Sistema (17) é mostrada nas Figs. 3.1(a) e 3.1(b), onde verificamos numericamente que para $\left.S_{w}^{-} \in\right] S_{w}^{+}, 1-S_{o r}$ ] o Problema de Riemann (1), (16) tem solução por onda de rarefação. Nestas figuras observamos que temos uma onda de rarefação conectando $\left(S_{w}^{-}, C_{s o}^{-}\right) \operatorname{com}\left(S_{w}^{+}, C_{s o}^{+}\right)$. 


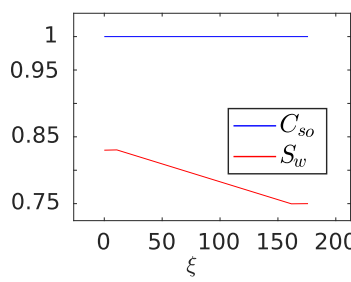

(a) $U^{-}=(0.83,1)$

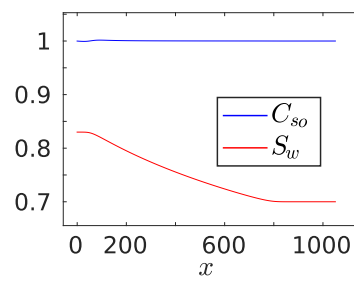

(b) $U^{-}=(0.83,1)$

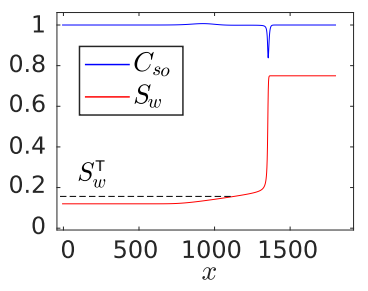

(c) $U^{-}=(0.12,1)$

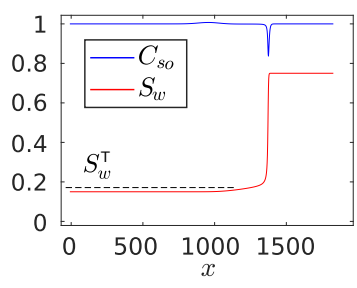

(d) $U^{-}=(0.15,1)$

Figura 3.1: Soluções do Sistema (1) com $U^{+}=(0.75,1)$ : (a)-(b) solução por ondas de rarefação e (c)-(d) solução por ondas compostas. Nas figuras (a) e (c) foi usado MATLAB, e em (b) e (d) foi usado RCD.

Com $U^{+}=(0.75,1)$ foi verificado que para $S_{w}^{-}=S_{w}^{T}, C_{s o}^{-}=1$ o estado $U^{-}$satisfaz a condição de Rankine-Hugoniot e satisfaz que a velocidade da característica $\lambda_{1}\left(U^{-}\right)$coincide com a velocidade de choque, veja Eq. (10). Neste caso dizemos que o choque é característico à esquerda e a solução do problema de Riemann consiste em um grupo de duas ondas sem estado intermediário constante entre elas. Tal grupo de ondas é chamada na literatura de "ondas compostas" [6]. Para $S_{w}^{-} \in$ $\left[S_{w c}, S_{w}^{T}\left[\right.\right.$, temos uma solução composta. Como exemplo, consideramos $U^{-}=(0.12,1)$ e $U^{+}=$ $(0.75,1)$. A solução do Problema de Riemann (1), (16) consiste de um grupo de duas ondas com um estado intermediário entre elas: primeiro tem-se uma onda de rarefação conectando $\left(S_{w}^{-}, C_{s o}^{-}\right)$ com $\left(S_{w}^{T}, 1\right)$ seguido de um choque característico à esquerda que conecta $\left(S_{w}^{T}, 1\right)$ a $\left(S_{w}^{+}, C_{s o}^{+}\right)$, ver Fig. 3.1(c). Similar resultado é mostrado na Fig. 3.1(d), com $U^{-}=(0.15,1)$ e $U^{+}=(0.75,1)$.

\section{Conclusões}

Consideramos o modelo que descreve o deslocamento de óleo com ajuda de um solvente miscível na fase aquosa imiscível. O principal objetivo foi determinar a existência da solução na forma de ondas viajantes do problema de Riemann. Além disso, mostramos numericamente as soluções do problema de Riemann para alguns exemplos.

\section{Agradecimentos}

Agradecemos à Coordenação de Aperfeiçoamento de Pessoal de Nível Superior - Brasil (CAPES) Código de Financiamento 001 e à Fundação de Amparo à Pesquisa do Estado de Minas Gerais (FAPEMIG). Também agradecemos ao Prof. Dr. Aparecido J. de Souza por suas contribuções ao concluir este trabalho.

\section{Referências}

[1] Lake, L. Enhanced oil recovery. Old Tappan, NJ: Prentice Hall Inc., 1989.

[2] Lake, L., Walsh, M. Applying fractional flow theory to solvent flooding and chase fluids. Journal of Petroleum Science and Engineering, 281-303, 1989.

[3] Lambert, W., Alvarez, A., Ledoino, I., Tadeu, D., Marchesin, D. e Bruining, J. Mathematics and Numerics for Balance Partial Differential-Algebraic Equations (PDAEs). JSComput, 1-56, 2020.

[4] LeVeque, R. Numerical methods for conservation laws. (Vol. 3.) Springer, Birkhuser, 1992.

[5] Lozano, L., F, Zavala, R., Q. e Chapiro, G. Mathematical properties of the foam flow in porous media. Computational Geosciences, (Vol. 25), 515-527, 2021.

[6] Matos, V., Azevedo, A.,V., Da Mota, J., C. e Marchesin, D. Bifurcation under parameter change of Riemann solutions for nonstrictlyhyperbolic systems. Zeitschrift für angewandte Mathematik und Physik (Vol. 66) 1413-1452, 2015. 